\title{
Orthodontic Force and Tooth Movement With and Without Occlusal Loads: 3D Analysis Using Finite Element Method
}

\author{
Jean Marc Retrouvey," and Allahyar Geramy ${ }^{2}$ \\ ${ }^{1}$ Division of Orthodontics, McGill University, Montreal, Canada \\ ${ }^{2}$ Orthodontics Department, Tehran University of Medical Sciences, Tehran, IR Iran \\ *Corresponding author: Jean Marc Retrouvey, Division of Orthodontics, McGill University, Montreal, Canada. E-mail: jean-marc.retrouvey@mcgill.ca \\ Received: 2015 November 3; Accepted: 2015 December 8.
}

\begin{abstract}
Background: Occlusal loads are always present though not widely considered in orthodontic treatments. Applied force systems are analyzed in detail and taught but their interaction with occlusal force is oftentimes ignored. Numeric evaluation of this combination by finite element method is the main goal of this research.

Objectives: The present study has implemented FEA to investigate the effect of orthodontic force application on the tooth-periodontiumalveolar bone system.

Materials and Methods: A3D model of a lower premolar was designed. The model contained cortical and spongy bone, PDL, and tooth. A $1.73 \mathrm{~N}$ force decomposed to $1 \mathrm{~N}$ in each plane axis was applied as a random levelling and aligning force to assess the von Mises stress produced at the mesial aspect of the PDL from cervical down to the apical area. At the second phase a 200-Newton intruding force vector was applied simulating the occlusal force. The effects of the combination force system were evaluated in the same area of the PDL.

Results: The maximum finding of von Mises for both stages were in found in the cervical area. This was almost $0.267 \mathrm{MPa}$ for the first stage and 2.27 MPa for the second stage.

Conclusions: Our findings show that the co-existence of heavy occlusal forces due to clenching or bruxism in our tooth-periodontiumorthodontic force system has a significant influence on the magnitude and location of the high stress areas.

Keywords: Occlusal Force, Orthodontic Force, Von Mises Stress, Finite Element Method
\end{abstract}

\section{Background}

Inclined planes on opposing teeth provide occlusal contacts and have a great influence on maintaining tooth position and mandibular stability. Considering the isolated pairs of antagonistic teeth, these forces increase progressively in a non-linear manner as the bite point moves posteriorly. According to the American sleep disorders association (ASDA) in 1990, 85 - 90\% of populations show a sleep bruxism habit which is a non-functional physiological phenomenon (1). According to Nishigawa et al. measuring nocturnal bite force during sleep in 10 subjects using the miniature strain-gauge transducers, the highest and mean amplitude of detected bruxism were 22.5 and $42.3 \mathrm{Kgf}$, respectively (2). It is suggested that the multidirectional functional load disrupts the continuous mechanical force of the fixed orthodontic appliance, rendering it intermittent (3-5). Maxillary molar behavior considering the occlusal contacts (6), evaluation of the articular disk elastic properties (7) and measurement of the maxillary central incisor displacements after the application of a given orthodontic force (8) are among other examples of studies that validate finite element approach to this subject.

Finite element analysis (FEA) is a numerical means of analyzing the transmission of forces and stress in struc- tural systems (9). This method is highly precise and used has been to analyze structural stress in engineering for years. In this method, equations are solved by computers to calculate desired parameters like stress and displacement on the basis of the physical properties of structures (10). This method has been adapted from the engineering to dental biomechanics.

\section{Objectives}

The present study has been designed to investigate the effect of the application of orthodontic forces on the tooth-periodontium-alveolar bone system considering the dynamic circumstances of a tooth under the influence of occlusal forces.

\section{Materials and Methods}

A 3D model of a lower premolar was designed based on Ash dental anatomy. The model contained cortical bone, spongy bone, PDL, and tooth. Tooth dimensions were considered as average. PDL was assumed to be $0.25 \mathrm{~mm}$ all around the root. SolidWorks 2006 (300 Baker Ave. Concord, Massachusetts 01742, USA) was selected for the modeling phase. Then the model was transferred to the ANSYS Workbench version 11.0 (ANSYS Inc. Soutpointe,

Copyright (C) 2015, Iranian Journal of Orthodontics. This is an open-access article distributed under the terms of the Creative Commons Attribution-NonCommercial 4.0 International License (http://creativecommons.org/licenses/by-nc/4.0/) which permits copy and redistribute the material just in noncommercial usages, provided the original work is properly cited. 
275 technology drive, Cononsburg PA 15317, USA) for the calculation. Material properties were considered as homogenous and were applied based on recent researches (Table 1). The model was meshed. The meshed model contained 19772 nodes and 10534 quadratic tetrahedron elements and 4051 contact elements. All nodes at the base of the models were restrained as the boundary condition so that all rigid body motions were prevented.

Two phases of loading were applied: During the first phase, a 1.73-Newton force, directed 45 degrees to the occlusal and vertical planes decomposed to $1 \mathrm{~N}$ at each plane in 3D space, was applied on the center of the occlusal surface. During second phase, a 200-N intrusive force was added to the previous phase. Von Mises stress was evaluated along a path of nodes starting at the cervical area of the mesial side of the root along the PDL down and ending at the apical area of the mesial side.

\section{Results}

The results show that the maximum stress (Von Mises Stress) occur at the cervical areas of both finite element models, and is equal to $0.26678 \mathrm{MPa}$ in the first phase, when only an orthodontic force was loaded to the premolar and is equal to $2.2729 \mathrm{MPa}$ in the second phase, when an occlusal force was added to the previous system. A decrease in the stress value is observed in the sub-cervical area in both phases (Figure 1 A and B) and (Figure $2 \mathrm{~A}$ and B).

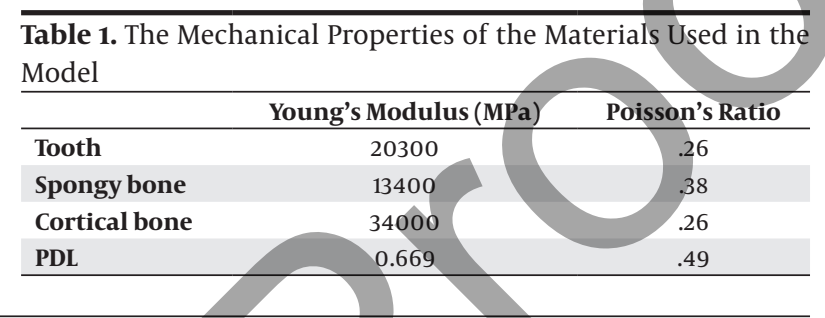

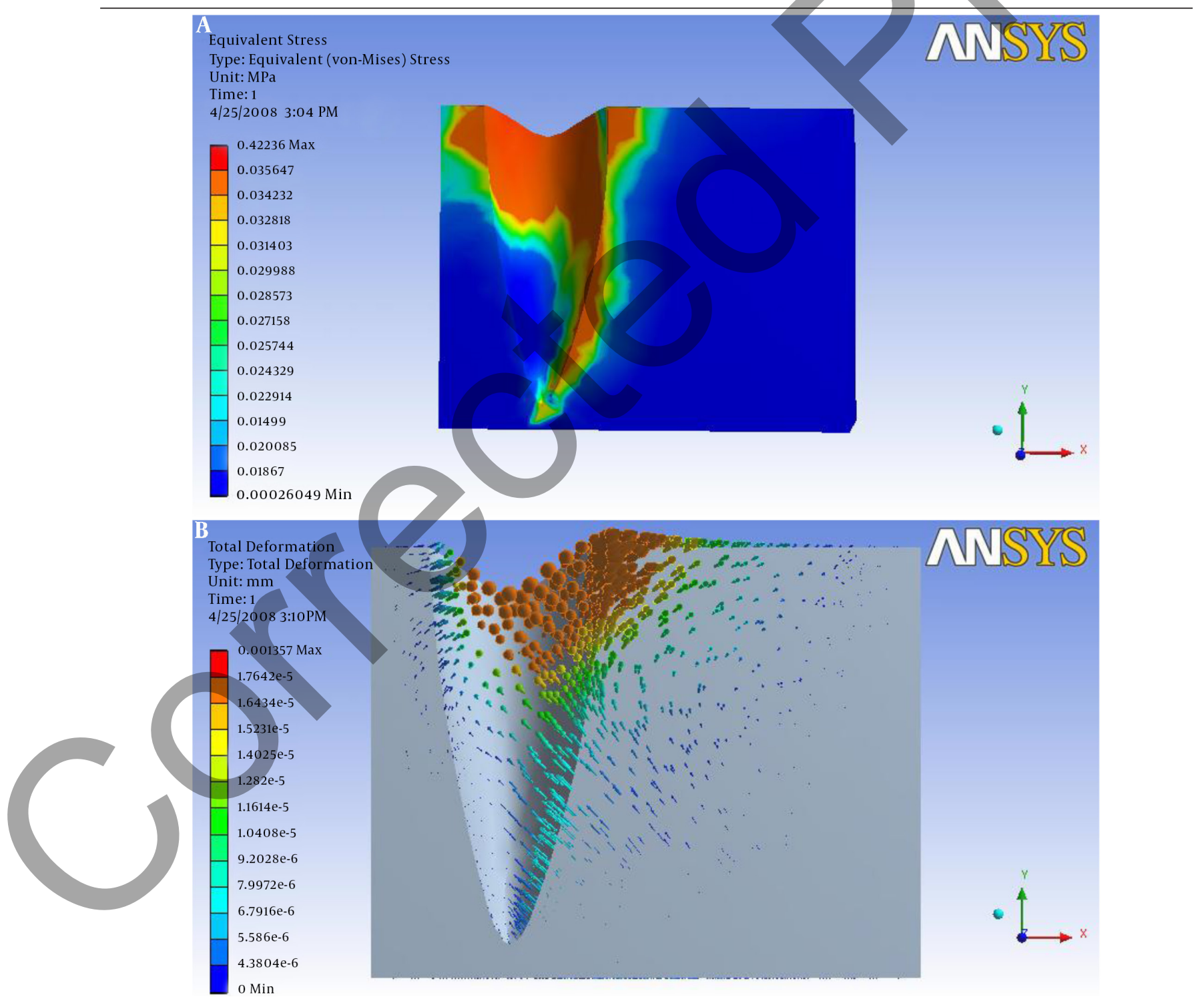

Figure 1. A, the Von Mises stress in the alveolar bone produced by the orthodontic force of $1 \mathrm{~N}$ decomposed into three components of $\mathrm{X}, \mathrm{Y}$ and $\mathrm{Z}$ (simulating buccal, mesial and occlusal directions) applied to the buccal surface of the premolar; B, The amount of deformation caused by this force. 

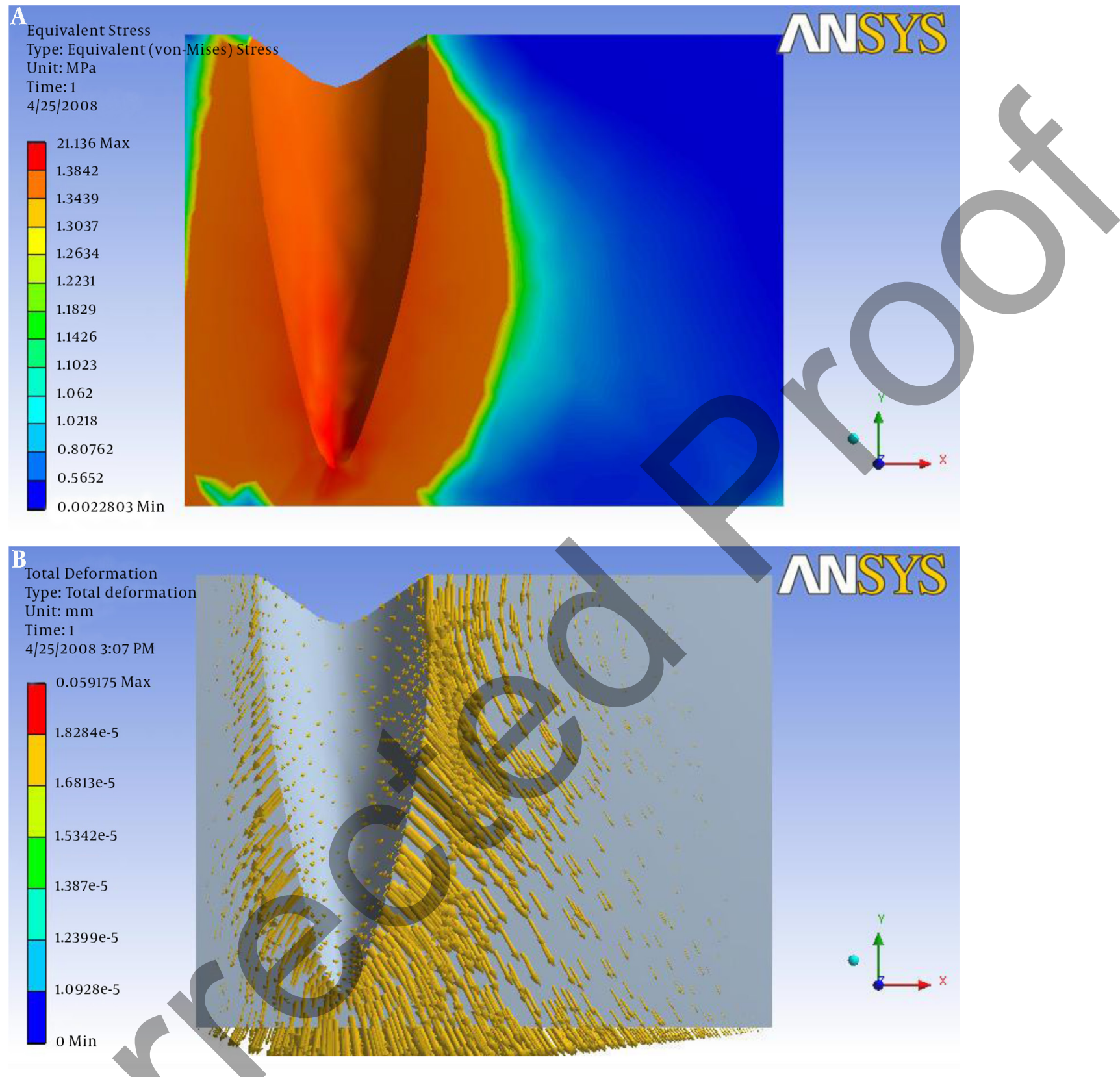

Figure 2. A, the Von Mises stress observed in the alveolar bone when orthodontic and occlusal forces were applied simultaneously; B, shows the amount of deformation in the alveolar bone as a result of application of the combination forces.

Moving toward the apical area, the results show a rather harmonic form of decrease in the first phase. As the minimum stress value that could be observed in the apical area, is equal to $0.040644 \mathrm{MPa}$ in the first phase and $0.80455 \mathrm{MPa}$ in the second phase.

\section{Discussion}

Occlusal contacts in the human dentition occur on inclined planes and have a great influence on maintaining tooth position and mandibular stability. When the occlusal system balance is disturbed, some negative effects may take place. When recorded between isolated pairs of antagonistic teeth, these forces increase progressively in a non-linear but monotonic manner as the bite point moves posteriorly (11).

It has been observed in a number of experimental situations, including the experiments described by Proffit and Sellers (12) that intermittent forces of appreciable magnitude quickly cause a small amount of intrusion, and then the teeth stabilize. Both in rodent incisors and in the experiments on human teeth, the period of quick intrusion ended after about 20 minutes. When the heavy force was released, there was a rebound also over about 20 minutes, followed by a continuation of eruption at a 
slower rate (12).

The PDL has a determinant influence on tooth instantaneous and short-term mobility, because of its much lower stiffness in comparison to the surrounding alveolar bone. This initial tooth mobility, which has to be distinguished from long-term orthodontic tooth movement, is affected by unique mechanical characteristics of the different components composing the PDL. However, the PDL influences also dental long-term movement, and its strain state regulates the activity of cells that are involved in the alveolar bone remodeling processes (13). Initial tooth mobility results from the damping effects found during short-term phenomena such as chewing or grinding, medium effects lasting several seconds to minutes during clenching and bruxism and long-term effects during the application of an orthodontic force system, and happen prior to the initiation of bone remodeling processes (14). It is suggested that the multi-directional functional load disrupts the continuous mechanical force system (3-5). Carano and Siciliani (5) compared the effects of continuous and intermittent forces on human fibroblasts. The fibroblasts, stimulated by a cyclical mechanical deformation (3 minutes stress, 3 minutes relaxation), produced 200 percent more collagenase in comparison with the control. When fibroblasts were deformed by a continuous distortion of the same amount as the cyclical one, the increment of collagenase production was lower (30 - 60 percent) in relation to the control. These results emphasize the importance of the modality of mechanical force application to cell cultures and suggest that the same differential behaviour could influence tissue response to mechanical stimuli. Our findings show that the co-existence of heavy occlusal forces due to clenching or bruxism in our tooth-periodontium-orthodontic force system has a significant influence on the location of the high stress areas and the location of the center of rotation. Putting the last two statements together we can conclude that heavy occlusal forces could be considered as influential factors in the orthodontic force- tooth system calculations. Many orthodontists consider using a bite plate or some fixed disclusion appliance for their patients as an adjunct to the orthodontic therapy to eliminate heavy occlusal loadings from the biomechanical system (15-17).

Amemori et al.(1) developed a three dimensional system to measure nocturnal mandibular movements by using a PIN photodiode sensor. The frequency and duration of the bruxing event were $4.5-10.9$ and $47.8-174.9$ seconds per hour, respectively. Clenching type bruxism was most frequently observed in their subjects and EMG activities during clenching were stronger than grinding. Etzel et al. (18) reported that $66 \%$ of nocturnal bruxism events were greater than the force of diurnal chewing. Rugh and Harlan (19) also concluded that the force of nocturnal bruxism might be more harmful than the equivalent forces during mastication because the contractions were often isometric, the intervals were longer and the teeth contacted in eccentric, unstable jaw positions (1).

In 2006, Gomes de Oliveira et al. (20) evaluated the ef- fects of different occlusal contact patterns on tooth displacement in an adult dentition using a three-dimensional FE model of a human maxilla and mandible. Small changes in the standard distribution of occlusal contacts resulted in an imbalance of occlusal forces and changes in dental positioning. All simulations showed mesial displacement of posterior teeth. The results of this study showed that small alterations in occlusal contacts commonly observed in prosthetic, orthodontic or restorative treatments could disrupt the balance of the occlusal system (20). Their findings are in accordance with our observations in some of our adult orthodontic patients, as they report periods of clenching and sleep bruxism at the onset of orthodontic therapy.

Results show an almost 10 fold increase in load at the cervical area when occlusal forces are added to the orthodontic system and an almost 20 fold increase when we compare the two force systems in the apical area. These forces systems are actually developing much more force than required for primary bone resorption and may promote sterile hyalinization of the periodontal ligament, increasing pain levels and potentially promoting root resorption

\subsection{Conclusion}

It can be concluded that heavy occlusal forces due to clenching and bruxism, although classified as intermittent forces, have the potential to interfere with the orthodontic force system and may delay the beginning of the tooth movement. Indeed, they are capable of triggering a cascade of fibroblasts activity in areas other than where we want the mechanical stress to be applied. Which is in the direction of the orthodontic forces.

\section{References}

1. Amemori Y, Yamashita S, Ai M, Shinoda H, Sato M, Takahashi J. Influence of nocturnal bruxism on the stomatognathic system. Part I: a new device for measuring mandibular movements during sleep. J Oral Rehabil. 2001;28(10):943-9. [PubMed:11737566]

2. Nishigawa K, Bando E, Nakano M. Quantitative study of bite force during sleep associated bruxism. J Oral Rehabil. 2001;28(5):48591. [PubMed: 11380790]

3. Terespolsky MS, Brin I, Harari D, Steigman S. The effect of functional occlusal forces on orthodontic tooth movement and tissue recovery in rats. Am J Orthod Dentofacial Orthop. 2002;121(6):6208. [PubMed:12080315]

4. Butler T. Comparative histologic study of heavy intermittent and light continuous forces. Am J Orthod. 1969;55(3):304-5. doi: 10.1016/0002-9416(69)90113-4. [PubMed: 4975045]

5. Carano A, Siciliani G. Effects of continuous and intermittent forces on human fibroblasts in vitro. Eur J Orthod. 1996;18(1):19-26. [PubMed: 8746174]

6. Magne P, Belser UC. Rationalization of shape and related stress distribution in posterior teeth: a finite element study using nonlinear contact analysis. Int J Period Restor Dent. 2002;22(5):425-34

7. Beek M, Koolstra JH, van Ruijven LJ, van Eijden TMGJ. Three-dimensional finite element analysis of the human temporomandibular joint disc. J Biomech.2000;33(3):307-16. doi:10.1016/S00219290(99)00168-2. [PubMed:10673114]

8. Jones ML, Hickman J, Middleton J, Knox J, Volp C. A validated finite element method study of orthodontic tooth movement in the human subject. J Orthod. 2001;28(1):29-38. doi: 10.1093/or- 
tho/28.1.29. [PubMed: 11254801]

9. Cattaneo PM, Dalstra M, Melsen B. The finite element method: a tool to study orthodontic tooth movement. J Dent Res. 2005;84(5):428-33. [PubMed:15840778]

10. Rubin C, Krishnamurthy N, Capilouto E, Yi H. Stress analysis of the human tooth using a three-dimensional finite element model. J Dent Res. 1983;62(2):82-6. [PubMed: 6571871]

11. Solberg WK, Rugh JD. The use of bio-feedback devices in the treatment of bruxism. J South Calif Dent Assoc. 1972;40(9):852-3. [PubMed: 4538306]

12. Proffit WR, Sellers KT. The effect of intermittent forces on eruption of the rabbit incisor. J Dent Res. 1986;65(2):118-22. [PubMed: 3455965]

13. Kawarizadeh A, Bourauel C, Zhang D, Gotz W, Jager A. Correlation of stress and strain profiles and the distribution of osteoclastic cells induced by orthodontic loading in rat. Eur J Oral Sci. 2004;112(2):140-7. doi: 10.1111/j.160 0-0722.2004.00116.x. [PubMed: 15056111]

14. Natali AN, Carniel EL, Pavan PG, Bourauel C, Ziegler A, Keilig L. Experimental-numerical analysis of minipig's multi-rooted teeth.
J Biomech. 2007;40(8):1701-8. doi:10.1016/j.jbiomech.2006.08.011. [PubMed: 17074355]

15. Timm TA, Ash MM. The occlusal bite plane splint. An adjunct to orthodontic treatment. J Clin Orthod.1977;11(6):383-90. [PubMed: 275270]

16. Sullivan TC. A new occlusal splint for treating bruxism and TMD during orthodontic therapy. J Clin Orthod. 2001;35(3):142-4. [PubMed:11314592]

17. Kessler M. The bite plate--an adjunct in periodontic and orthodontic therapy. J Periodontol. 1980;51(3):123-35. doi: 10.1902/ jop.1980.51.3.123. [PubMed: 6988569]

18. Etzel KR, Stockstill JW, Rugh JD, Fisher JG. Tryptophan supplementation for nocturnal bruxism: report of negative results. $J$ Craniomandib Disord.1991;5(2):115-20. [PubMed: 1812137]

19. Rugh JD, Harlan J. Nocturnal bruxism and temporomandibular disorders. Adv Neurol.1988;49:329-41. [PubMed:3278546]

20. Gomes de Oliveira S, Seraidarian PI, Landre JJ, Oliveira DD, Cavalcanti BN. Tooth displacement due to occlusal contacts: a threedimensional finite element study.J Oral Rehabil. 2006;33(12):87480. doi:10.1111/j.1365-2842.2006.01670.x. [PubMed:17168929]
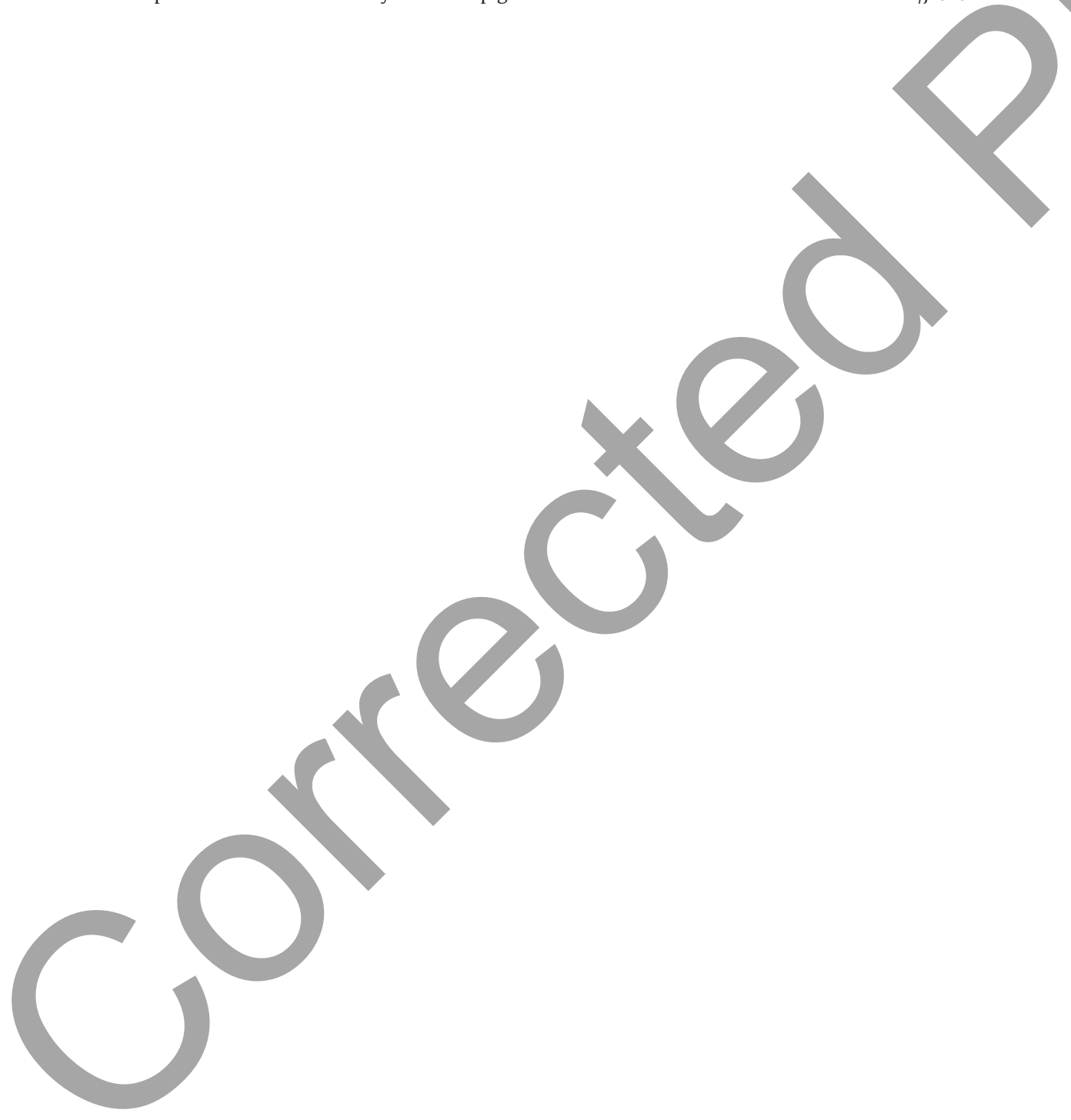